International Journal of

Advanced Science and Convergence

ISSN : $2619-8150$

\title{
Applying MQTT Protocol and Node-Red Programming in Designing IoT based Home Automation
}

\author{
Oscar Bryan M. Magtibay
}

Electrical Engineering Department, College of Information Technology and Engineering, De La Salle Lipa, 1962 J.P. Laurel Highway, Lipa City Batangas Philippines 4217

\begin{abstract}
Background/Objectives: Nowadays, wireless sensor and control are integrated with the Internet Protocol (IP) to develop the Internet of Things (IOT) for connecting devices which are used in everyday life to the internet. Methods/Statistical analysis: Users can now monitor and control different devices even they are not at home provided there is internet access. The primary advantages from this concept include wireless devices being a part of a cloud-based system by which, it can to interact and communicate, maximize safety, comfort, convenience and energy-savings. Existing systems require additional communication hardware (such as LAN, Bluetooth and RF) and proprietary software to the system which increases to the cost of the system. Findings: There is a need for easy deployable solutions by using open source solutions for both hardware and software. This paper proposes the development of the hardware using ESP8266 microcontroller, the use of MQTT protocol as means of sending and receiving messages from the wireless devices to a central server and the use Node-red Programming for creating the web based application for the loT based Home Automation. A comparison with existing home automation system was made to evaluate the effectiveness of the system. Improvements/Applications: The use of NodeMCU as the microcontroller equipped with the MQTT protocol and uses a Raspberry $\mathrm{Pi}$ as the MQTT broker reduces the need for additional hardware such as Bluetooth and Zigbee and use the existing WiFi netrok at home to control appliances and monitor the power consumption at home.
\end{abstract}

\section{Index Terms}

Automation, ESP8266, Internet of Things, MQTT, Node-Red

Corresponding author : Oscar Bryan M. Magtibay

oscar.bryan.magtibay@dlsl.edu.ph

- Manuscript received July 15, 2019.

- Revised August 17, 2019 ; Accepted September 20, 2019.

- Date of publication September 30, 2019.

(C) The Academic Society of Convergence Science Inc.

2619-8150 @ 2019 IJASC. Personal use is permitted, but republication/redistribution requires IJASC permission. 


\section{INTRODUCTION}

\section{A. Background}

The internet of things, or IoT, is a system of connected digital devices and machines, objects, animals or people that are provided with unique identifiers (UIDs) and the ability to transfer or receive data over a network without requiring human intervention. [1]

IoT's objective is to connect human, physical object environments, and internet into one. Through IoT, people can create a device that can monitor, analyze, control, and automate. [2] Today, appliances and smart devices can connect to the internet and create decisions at home.

A Building Energy Management System consists of software and hardware. The hardware component includes different sensors and switches. The interconnection of all sensors, switches and devices is by means of transferring data in and out through a server in a real-time manner using a software application. It is essential to have good communication connectivity of all components integrated into one building management system for easy, and interactive control. [1]

The concept of home automation has adapted by researchers, electronics hobbyist, and the consumers and has grown and developed throughout the years. Developments have been made to the design for home automation. Such existing systems are based on Bluetooth and RF communication and using different controllers like Arduino and PIC. The study of Mowad et.al.[3] discusses on using smartphones to control and monitor the security at home. Althobaiti et. al. [4] focuses on WiFi communication in which the user uses his smartphone or PC to connect to a server where the Arduino microcontroller is connected via Serial communication. By this, the user can control the appliance at home. The study of David et. al. [5] uses both internet and Bluetooth to control appliances within or far away from home. They use Http protocol to connect from the Arduino microcontroller with $\mathrm{WiFi}$ module that serves as a micro-server where the smartphone or PC could connect. Hasan et.al. [6] uses ATMEGA16 controller, Bluetooth control appliances and GSM module to monitor the security at home.

MQTT is a machine-to-machine (M2M)/"Internet of Things" protocol. It was made as a lightweight publish/subscribe messaging transport. It is useful for connections with remote locations where a small code footprint is required and/or network bandwidth is at a premium. [7] For example, it has been used in sensors communicating to a broker via satellite link, over occasional dial-up connections with healthcare providers, and in a range of home automation and small device scenarios.

Node-RED is a flow-based programming tool, originally developed by IBM's Emerging Technology Services team and now a part of the JS Foundation. Invented by J. Paul Morrison in the 1970s, flow-based programming is a way of describing an application's behavior as a network of black-boxes, or "nodes" as they are called in NodeRED. Each node has a well-defined purpose. It is given some data, it manipulates and passes that data between the nodes. [8]

\section{B. Objective of the Study}

Existing systems require additional communication hardware (such as LAN, Bluetooth and Zigbee) and proprietary software to the system which increases to the cost of the system. There is a need for an easy deployable solution by using open source solutions for both hardware and software.

In line with this, the researcher applies the use of MQTT protocol and Node-red programming in designing IoT based Home Automation. Specifically, it aims to achieve the following: to determine the user and hardware requirements for the specifications of the system; design the system architecture, schematic diagrams and layouts; assemble and deploy the hardware; apply MQTT protocol and Node-red application that will be the interface to control the appliance and monitor the power consumption of the room; and test and evaluate the results based on existing systems.

\section{Scope and Limitations}

The study for IoT-Based Home Management System covers the design and development of the hardware and software application for the data acquisition of energy consumption and controls. It will be limited to the number of appliances it can control and monitor. The hardware device will be connected to switches of lamps and outlets for small appliances. The rating of the device is limited to the rating of the relay and current sensor to be used for the system. It will utilize the existing $\mathrm{WiFi}$ connection to communicate the devices to the server/ broker.

\section{RELATED Literature}

The study of Gupta et. al., Taking MQTT and NodeMcu to IOT: Communication in Internet of Things, uses MQTT protocol for data transmission. It is a publisher and subscriber based protocol which 
allows multiple devices to communicate with each other over a wireless network. The top most layer of TCP/IP is Application layer and MQTT protocol is used in this layer. Figure 1 show the system of the MQTT protocol. [9]

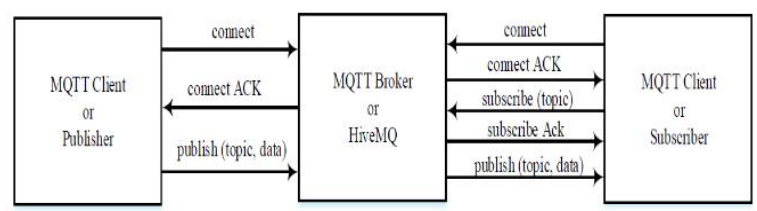

Fig. 1. MQTT System

The MQTT connection has types of operators: the MQTT client and the MQTT broker or server. The information sent by MQTT is called as application message. When the MQTT client must establish a connection to the broker in order to publish or subscribe to an information or a topic, an acknowledgment must be made between the client and the broker in order to publish or subscribe.

This paper focused on the execution of Arduino using Serial protocol and, NodeMCU using MQTT protocol. It describes the different possible service models for communication among the IoT devices. It discusses the communication between client and server using wireless (Wi-Fi) and wired (USB) transmission medium. MQTT protocol is the main focused area in this work. The protocol was test by using publish and subscribe message on the same and different machine, and a microcontroller with built-in wireless communication such as a Node MCU is set up, and a Raspberry Pi that serve as the server/ broker. It was found that it has slower transfer speed compared to a wired connection but the reliability of data is stable as long as the $\mathrm{Wi}-\mathrm{Fi}$ is available.

Jaloudi's MQTT for IoT-based Applications in Smart Cities, presents a study on the use of the MQTT communications protocol for the Internet of Things in Smart City applications. They use inexpensive components such as Arduino, ESP8266 and Raspberry Pi in the creation of an application for Smart City. MQTT protocol was used because of its lightweight, easy to program, flexibility and reliability. Based on the study, the MQTT is suitable for small-to-medium IoT-based business applications of Smart Cities that use cloud-based servers and for medium-to-big business applications that rely on LANs and Intranet. For example, Smart City applications such as energy monitoring, smart buildings, home automation and smart healthcare system may employ the MQTT protocol in all levels of communications. [10]

The study of Morocco et.al., Industrial
Communication Based on Modbus and Node-red, uses Modbus protocol to communicate industry based sensors and controller to a single on-board computer and wirelessly connect to the wireless network through the use of MQTT and Node-RED application. By this, Internet of Things (IoT) and Cyber Physical Systems (CPS) have change the industrial world with the introduction of Industry 4.0 technology to create smart factories. [11]

The study of Hajovski et. al. describes the implementation of wireless communication between the control unit and the remote device using IQRF technology. With the use of Node-RED programming, it creates reliable and easy to use application for the Internet of Things. The result of the study is that it can measure physical quantities of temperature, light intensity and value of a potentiometer. The entire implementation runs on the Raspberry Pi platform and on the IQRF technology, which provides the wireless communication within the IQMESH network. [12]

The study of Yokotaini et. al. on Comparison with HTTP and MQTT on Required Network Resources for IoT discusses the advantages of and MQTT network over Http on the rate of sending and receiving transmission to devices due to its lightweight in nature of publishing and subscribing to a particular topic which the Http does not have. [13]

\section{METHODOLOGY}

\section{A. System Architecture}

The home automation system uses cloud-based public MQTT broker and a local MQTT broker to control the appliances both local or the internet.

Figure 2 shows the System Architecture of the System

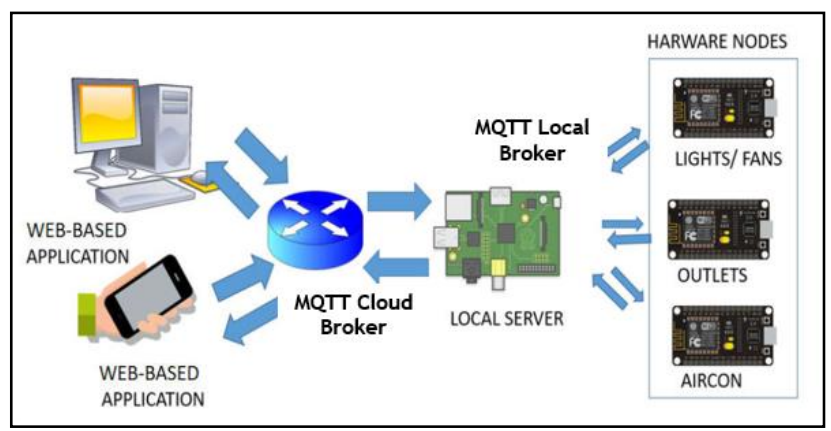

Fig. 2. System Architecture

The hardware nodes, composed of sensors, relays and microcontroller, will acquire data on power from the lights fans and will serve as the switch for the said appliances. Those data are transmitted using 
Cloud Network that will be passed to the local server using the MQTT protocol. The software application will be based on the Node-RED programming tool. The application displays the power consumption as well as the remote control for the appliances.

\section{B. Hardware Components Used}

- Microcontroller. NodeMCU was used to acquire the current values, control the switches and connect to the network through WiFi.

- $\quad$ Current sensor. ACS712 current sensor was selected to measure the current by using the concept of Hall effect.

- $\quad$ Relay module. Single channel relay module was used to switch on and off the appliances.

- Power Supply. Hi-Link 5V DC power supply was used for low power application suited for microcontroller use.

\section{Hardware Circuit Design}

Figure 3 shows the connection diagram of the hardware.

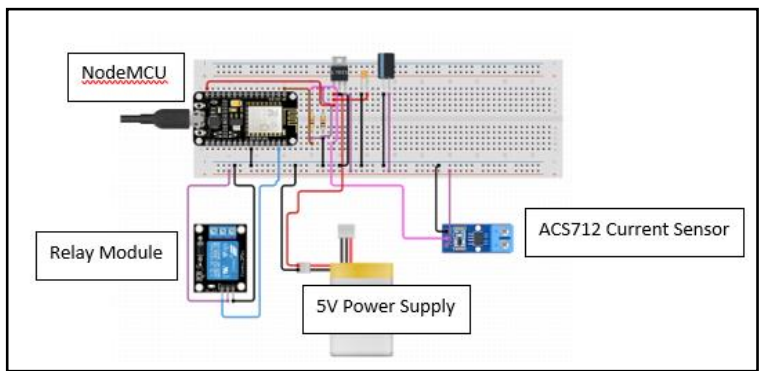

Fig. 3. Connection Diagram of the Control Node

The current sensor is connected to one line of the outlet to measure the current. The power supply used is 5 volts and is used to power the NodeMCU. The output of the sensor is connected to the analog $\mathrm{A} 0$ of the NodeMCU. The relay module is connected to the pin D2 of the microcontroller. The NodeMCU microcontroller is responsible for acquiring the data from the sensor controls the appliances using WiFi communication.

\section{MQTT Protocol Setup}

The MQTT protocol was used as the communication between different hardware nodes. Two MQTT brokers was used to control the devices at home or away, the public broker at iot.eclipse.org and a local broker using Raspberry $\mathrm{Pi}$ with a mosquito broker installed. The phone or PC used to control and monitor the devices are the MQTT clients that will access the public broker. The Raspberry Pi which is located locally, will then subscribe to the topics with in the public broker. Once the information was sent by the client, the local server will get the information then will publish the message for the subscribe node hardware to receive the necessary information to open or close the appliance.

\section{E. Web Application Design}

The web application is done using Node-Red. It is a programming tool that uses nodes that are interconnected to create a graphical user interface program that can be accessed through the web browser such as Google Chrome or Internet Explorer.

Figure 4 shows the Node-RED programming environment.

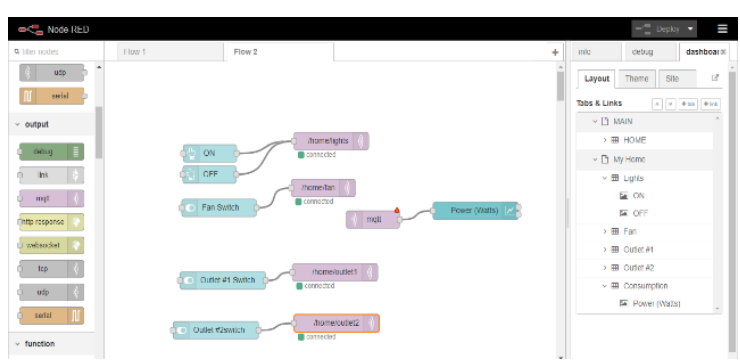

Fig. 4. Node-red Programming Environment

It gives a browser-based environment that makes it easy to wire together flows using the number of nodes in the palette that can be created to its runtime in a single-click. The dashboard node serves as the foundation for the graphical use interface of the web application. The MQTT node input/ output node is the subscribe and publish function for the Node-RED to monitor the power consumption and to send controls to each device.

\section{Test AND RESUlts}

The testing is done in one room to test the wireless connectivity and power consumption of the hardware (idle condition) used.

The researcher recorded the power consumption of the Raspberry Pi server and the Node-MCU during operation.

Figure 5 shows the power consumption used for both the control node and the server.

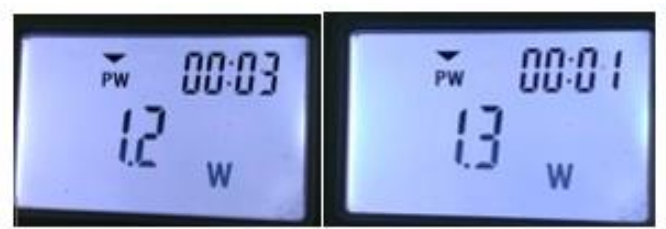

Fig. 5. Consumption of the hardware node (idle) and server 
The consumption of the control node is 1.2 watts while the server which is a Raspberry Pi Zero $\mathrm{W}$ is 1.3 watts.

The web application has a graphical user interface that shows the controls and the hourly power consumption at home.

Figure 6 shows the Web Application of the home automation

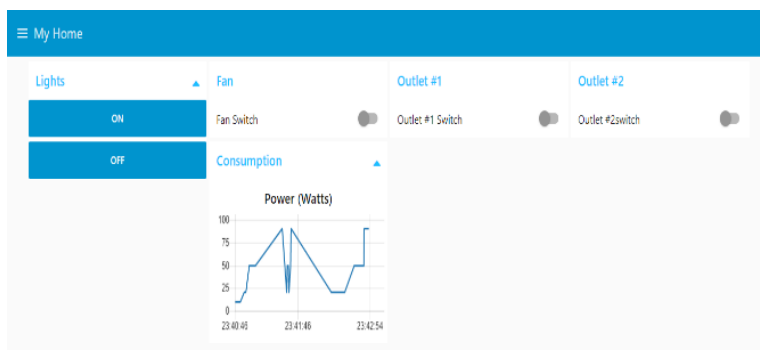

Fig. 6. Web Application

The web application can be accessed through the use of any web browser in different Operating systems (Windows, Mac OS, Android, iOS).

Figure 7 shows the connectivity of the web application through Twitter and Facebook.

Using the Twitter node in Node-RED, the system can notify the user through Twitter or Facebook of the power consumption depending on the set time and intervals.

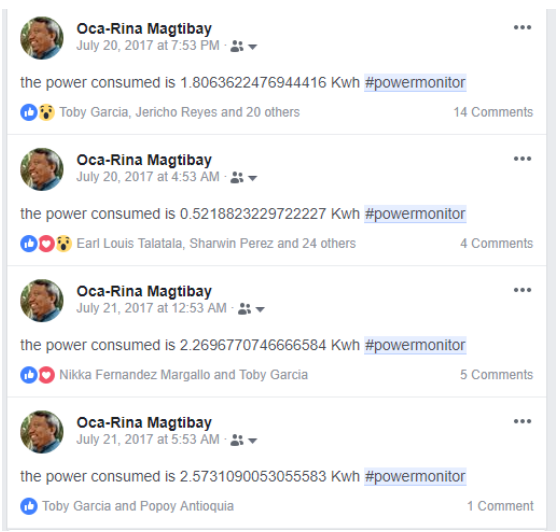

Fig. 7. Connection test through the internet using Facebook/ Twitter

The Twitter node accesses a user account and tweets the power consumption at certain intervals. The user then notifies on Facebook through Twitter Facebook integration.

To validate the solution, a table was made to compare existing systems based on the project.

Table 1 shows the comparison.
Table 1. Comparision of existing systems to the project

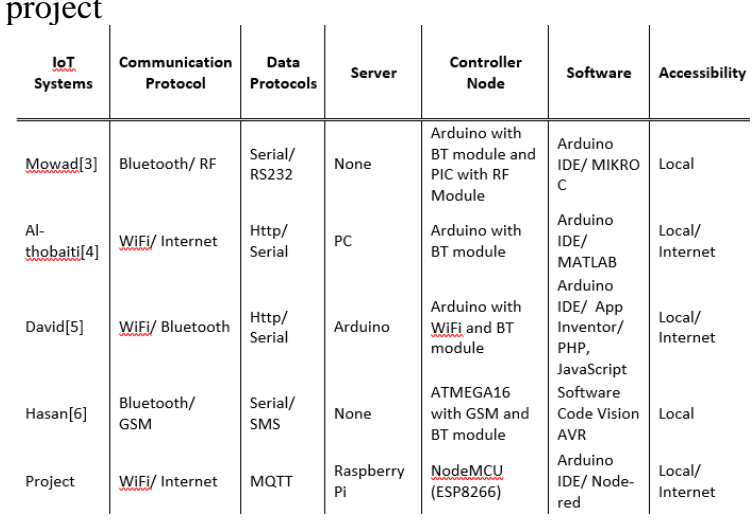

Existing systems use Bluetooth that limits the systems maneuverability to control outside the home. Each device must be paired to the smartphone in order to control the appliances. Others use a PC server that consumes at least 200 watts of power which is high considering controlling appliances in a home setting. Using Arduino as an Http webserver restricts the flexibility to additional functions such as database due to its limited memory. It could be seen that the use for a single data protocol such as MQTT makes the system simple and efficient most specially for microcontroller. The use of Node-red provides a web-based user interface that is easy to use and needs no installation of application for both smartphones and PC.

\section{CONCLUSION}

Based on results, the following conclusions are drawn: the selection of the hardware components meets the needed specifications to measure the parameters and control the appliances; the system architecture was made to simplify the system and determine the hardware and software used in the system. The use of MQTT protocol and Node-RED application was suitable for developing IoT systems at home since it gives ease of use and flexibility as compared with the existing IoT Home Automation Systems.

\section{REFERENCES}

[1] HOW A BUILDING MANAGEMENT SYSTEM WORKS (2018). Retrieved from http://advancedcontrolcorp.com/blog/2017/01/how-buildingmanagement-system-works/

[2] Wibobo F.W. (2018). A Low-Cost Home Automation System Based-On Internet of Things, Retrieve from https://www.researchgate.net/publication/320191780_A_Lo w-Cost_Home_Automation_System_Based On_Internet_of_Things

[3] Mohamed Abd El-Latif Mowad, Ahmed Fathy \& Ahmed Hafez. (2014). Smart Home Automated Control System Using Android Application and Microcontroller, 
International Journal of Scientific \& Engineering Research, 5(5), 2014.

[4] Bader M. O. Al-thobaiti, Iman I. M. Abosolaiman, Mahdi H. M. Alzahrani, Sami H. A. Almalki \& Mohamed S. Soliman. (2014). Design and Implementation of a Reliable Wireless Real-Time Home Automation System Based on Arduino Uno Single-Board Microcontroller, International Journal of Control, Automation and Systems, 3(3).

[5] Nathan David, Abafor Chima, Aronu Ugochukwu, Edoga Obinna. (2014). Design of a Home Automation System Using Arduino, International Journal of Scientific \& Engineering Research, 6(6).

[6] Raqibull Hasan, Mohammad Monirujjaman Khan, Asaduzzaman Ashek, Israt Jahan Rumpa, Microcontroller Based Home Security System with GSM Technology, Open Journal of Safety Science and Technology, 2015

[7] What is MQTT. (2019). Retrieve from www.mqtt.org

[8] Node-RED: Flow-based programming for the Internet of Things, Retrieve from http://nodered.org, 2019

[9] Kashyap, M., Sharma, V. \& Gupta, N. (2018). Taking MQTT and NodeMcu to IOT: Communication in Internet of Things, International Conference on Computational Intelligence and Data Science (ICCIDS 2018).

[10] Jaloudi S. (2019). MQTT for IoT-based Applications in Smart Cities, PJTAS, no.2.

[11] Mohamed Tabaa*, Brahim Chouri, Safa Saadaoui \& Karim Alami. (2018). Industrial Communication based on Modbus and Node-RED, The 9th International Conference on Ambient Systems, Networks and Technologies.

[12] Hajovsky R., Pies, M. \& Skovranek Jiri. (2018). Use of the IQRF and Node-RED technology for control and visualization in an IQMESH network, International Federation of Automatic Contol (IFAC)

[13] Tetsuya Yokotani \& Yuya Sasaki. (2016). Comparison with HTTP and MQTT on Required Network Resources for IoT The International Conference on Control, Electronics, Renewable Energy and Communications. 